\title{
EL FUNDAMENTO METAFÍSICO DE LAS TEORÍAS ADMINISTRATIVAS
}

\author{
"All men by nature desire to know" \\ "Metaphysics". Aristóteles
}

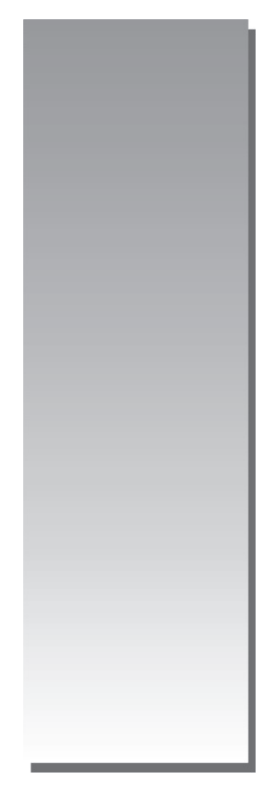

\author{
Augusto Hidalgo Sánchez ${ }^{*}$ \\ augustohidalgo@hotmail.com
}

\begin{abstract}
RESUMEN
La ciencia de la administración ha surgido hace unos 100 años a partir de propuestas teóricas de inspiración normativa. A estas teorías se fueron agregando otras de naturaleza sociológica y psicológica. Luego de la Segunda Guerra Mundial, los progresos de las matemáticas generaron un gran esfuerzo en los teóricos de la administración para buscar su adaptación a la solución de los problemas de las organizaciones y de su entorno. Últimamente, una nueva corriente de pensamiento llamado sistémico está generando no solamente una nueva serie de tipos de negocios sino de enfoques para la solución de los problemas organizacionales. Por lo tanto, se puede resumir que cuatro son las fuentes de pensamiento metafísico de la ciencia de la administración: normativa, sociológica, matemática y sistémica. Se sugiere que los protocolos y reglamentos de investigación académica en administración en las universidades incorporen los proyectos de investigación no cuantitativos y que se ciñan a enfoques epistemológicos alternativos.
\end{abstract}

Palabras claves: Metafísica, epistemología, ciencia de la Administración, teoría, modelo.

\begin{abstract}
The management science emerged one century ago thanks to pioneer models of normative inspiration. Later, new theories of sociological and psychological nature were added to that body of knowledge. After de Second World War, the uprising of the applied mathematics in business improved the management of the organizations and their environment. Lately, a new stream of systemic nature is generating not only a new kind of business model but of business thinking. In sum, it is possible to state that there are four sources of administrative metaphysics: normative, sociological, mathematical and systemic. We suggest that the research procedures in universities should change in order to incorporate to the traditional quantitative approach, new qualitative ones born from the fountain of the three other metaphysics.
\end{abstract}

Keywords: Metaphysics, Epistemology, Management Science, Theory, Model, Metaphysical Foundation.

\footnotetext{
Bachiller en Ciencias Económicas y Licenciado en Administración de la Universidad Nacional de Trujillo, Perú. Magister en Administración de ESAN, Perú. Master of Science de The University of Tennessee en Knoxville, USA. Doctor en Administración de la UIGV, Perú. Profesor del curso de Teorías Administrativas por 21 años. Docente universitario por 35 años. Profesor Asociado de la Facultad de Ciencias Administrativas de la Universidad Nacional Mayor de San Marcos. Director de la Escuela de Administración de Negocios Internacionales de la Universidad Nacional Mayor de San Marcos. Ex - profesor Principal de la Universidad Nacional de Trujillo. Profesor de postgrado de: UNMSM, U. N. de Trujillo, U.N. del Centro del Perú (Huancayo). Ex gerente general del Gobierno Regional de La Libertad, ex - Gerente Regional de EsSalud. Ex gerente de empresas varias del sector privado. Consultor Senior en proyectos de fortalecimiento institucional para proyectos del BID, GTZ, Ministerio de Educación del Perú, entre otros.
} 


\section{INTRODUCCIÓN}

La administración es una actividad mental consustancial con la tarea natural de organizar las ideas para actuar, y para controlar los efectos de los resultados que nuestros actos producen. Consecuentemente y por muchos siglos, administrar no parecía una actividad digna de ciencia, a pesar que Jenofonte (1963) en su "Economía" o gobierno doméstico, ya la insinuaba. Naturalmente la política, como la construcción del futuro de los pueblos y como la tarea de administración más compleja, durante toda la historia siempre fue motivo de pensamiento agudo, allí tenemos a la "La República" de Platón. Aunque en realidad, desde la aurora de las ciencias, la política siempre ha estado separada de la administración.

Fue a mediados del siglo XIX cuando la primera revolución industrial se materializó en inmensas organizaciones empresariales industriales, cuando el utilizar solamente el sentido común de sus propietarios para administrar colapsó ante las gigantescas demandas. Aquellas organizaciones que aprendieron a sistematizar individual o colectivamente su propia experiencia, aquellas que aprendieron a comprender al ser humano y al entorno, fueron las que sobrevivieron y surgieron. Este es el caso por ejemplo de Western Union (2012), una empresa de telegrafía fundada en el año 1851 y que 162 años después se mantiene viva y exitosa.

A inicios del siglo XX, propietarios, ingenieros, y administradores por delegación de los dueños, tanto en Estados Unidos como en Europa, comenzaron a inquirir en los antecedentes de la emergente disciplina, así como en otras disciplinas ya establecidas del siglo XIX, y a sistematizar y a documentar sus propios hallazgos sobre este nuevo cuerpo del conocimiento. Los primeros administradores eficaces en dicho propósito fueron los ingenieros Frederick Taylor (1856 - 1915), norteamericano, y Henry Fayol (1841-1925), francés, quienes propusieron los primeros lineamientos generales de lo que sería la ciencia de la administración. Fue Fayol, en particular, quien propuso las funciones empresariales, las funciones administrativas y los principios de la administración. Por su parte, a las aplicaciones de Taylor se debió el boom de la economía norteamericana de antes de la Primera Guerra Mundial. Les siguieron una pléyade de ingenieros especializados en la planificación y el control. A esta etapa podríamos llamar el primer impacto sobre la administración.

Un segundo impacto se registró con la propuesta de la Teoría Burocrática por el sociólogo alemán Max Weber (1864-1920), quien formuló un conjunto de principios agrupados en el "modelo de burocracia" que configuraron "el modelo ideal" de organización, la misma que estaba diseñada para durar eternamente.

El tercer gran impacto en la naciente disciplina la generó el psicólogo Elton Mayo (1880- 1949), australiano, de la Universidad de Harvard, quien planteó la gran teoría de las Relaciones Humanas. Detrás de él, un gran número de seguidores afianzó el conjunto de teorías del comportamiento humano en las organizaciones. Mención gloriosa merecen los norteamericanos Philip Mc Gregor, Chester Barnard y Abraham Maslow (1981) con su "escala de las necesidades humanas".

El cuarto impacto tuvo como origen el escenario de la Segunda Guerra Mundial. La colosal producción de aviones, embarcaciones, tanques y demás vehículos de combate, aprovisionamientos y hombres no encontraban suficientes aeropuertos, centros de reabastecimiento, y personal especializado, de tal manera que fue necesario formular un conjunto de herramientas de tipo matemático que permitieran manejar estas complejas variables de manera efectiva y eficiente. Así surgieron las teorías cuantitativas de la administración, sin las cuales es inimaginable manejar los recursos de la civilización humana en su estado actual de desarrollo.

Un quinto impacto ocurrió a partir de los años 50 con la adopción de la Teoría General de Sistemas, y su hijo tecnológico, la computadora. El sexto y actual impacto tiene que ver con la necesidad de adaptación de las organizaciones a un ambiente globalizado por redes mundiales de fibra óptica, satélites y redes sociales, además de considerar los cambios en la dinámica económica mundial y los cambios en la ecología. Un conjunto de teorías denominadas contingentes están en plena ebullición tratando de responder las actuales demandas. 
De todo este conjunto de impactos, tal vez el hecho más sorprendente y verificable es que la naturaleza de la génesis de la gran mayoría de teorías administrativas no ha seguido únicamente el proceso epistemológico estandarizado, en el sentido clásico de que para probar la verdad de una proposición se debe seguir un proceso demostrativo problema-hipótesis-(proceso de verificación matemática)-ley. En muchos casos las teorías administrativas nacieron como producto de ejercicios de metafísica pura; otras emergieron como modelos heurísticos de tipo proposicional o "normativo", buscando estandarizar las conductas individuales y optimizar la toma de decisiones en organizaciones diversas.

Curiosamente, la investigación científica universitaria escolástica masiva que ha venido acompañando al desarrollo de la ciencia de la administración desde su aparición formal en los años 20 , se ha concentrado mayormente en el desarrollo y fortalecimiento de disciplinas complementarias como marketing, investigación de marketing, finanzas y contabilidad gerencial, logística y procesos de adquisiciones, transporte y almacenamiento, sistemas de información gerencial, sistemas de calidad, herramientas de la motivación, y control de los recursos humanos.

Finalmente, y como hecho curioso, en la mayoría de los principales journals o literatura especializada de investigación en administración no se evidencian esfuerzos por conducir a las teorías centrales primigenias de la ciencia de la administración (Fayol, Taylor, Weber) a las rigurosas pruebas epistemológicas con el objeto de demostrar si son verdaderas o no, eso probablemente porque no es tarea fácil o sencilla.

\section{Objetivo y métodos del estudio}

El objetivo del presente artículo es hacer un recuento de las principales teorías de la ciencia de la administración y explicar brevemente su contenido tomando como elemento central a la metafísica en sus procesos de génesis y formulación. El presente estudio, siendo exploratorio, solo busca promover el intercambio de ideas al respecto. No contiene hipótesis alguna ya que es un estudio bibliográfico que se propone identificar y definir conceptos relacionados con la génesis de las teorías de la administración. Un objetivo más ambicioso -pero muy inseguro- es ensayar una explicación del motor que da origen a las teorías administrativas. Este motor lo denominamos "metafísica de la administración", de la cual proponemos su composición.

Para esto se ha revisado la literatura que incluye algunas fuentes filosóficas, pasando en el intermedio por las principales teorías mainstream de la ciencia de la administración generadas desde 1915, incluyendo algunas tecnologías administrativas. También se han revisado los planteamientos de los metodólogos en administración y en ciencias sociales, particularmente de los latinoamericanos.

\section{RESULTADOS}

Visión general de la concepción filosófica de la ciencia de la administración

La ciencia de la administración es una disciplina compleja, mezcla de ciencia, técnica, arte y experiencia, que tiene en su acervo una gran variedad de problemas, conceptos, categorías, principios, teorías y tecnologías, cuya génesis está indistintamente vinculada tanto al enfoque cuantitativo como al cualitativo de la ciencia. El amplio análisis de Hernández Sampieri et. al. (2010) sobre los enfoques de la ciencia en general, expuesto en la Tabla $\mathrm{N}^{\circ} 1$, parece describir con mucha aproximación a la ciencia de la administración.

\section{Qué es la metafísica}

Es la quinta esencia del razonamiento. Es la rama de la filosofía que se encarga de estudiar la naturaleza, estructura, componentes y principios fundamentales de la realidad. Aborda problemas centrales de la filosofía como los fundamentos de la estructura de la realidad, el sentido, y finalidad última de todo ser, todo lo cual se sustenta en el llamado principio de no contradicción. La metafísica tiene como tema de estudio la ontología, o ciencia que estudia el ser en tanto que ser. Su énfasis no está en probar algo sino en construir modelos.

La metafísica es esencialmente intuición, un despertar de la razón. Es la luz frente al problema. Es el primer arranque de interpretación y de 
Cuadro $\mathrm{N}^{\circ} 1$ Los enfoques de la ciencia

\begin{tabular}{|c|c|c|}
\hline Dimensiones & Enfoque Cuantitativo & Enfoque Cualitativo \\
\hline $\begin{array}{l}\text { Marcos generales de } \\
\text { referencia básicos. }\end{array}$ & Positivismo, neopositivismo, postpositivismo. & Fenomenología, constructivismo, naturalismo, interpretativismo. \\
\hline Punto de partida. & $\begin{array}{l}\text { Hay una realidad que conocer. Esto puede } \\
\text { hacerse a través de la mente. }\end{array}$ & Hay una realidad que descubrir, construir e interpretar. La realidad es la mente. \\
\hline Realidad a estudiar. & $\begin{array}{l}\text { Existe una realidad objetiva única. El mundo } \\
\text { es concebido como externo al investigador. }\end{array}$ & $\begin{array}{l}\text { Existen varias realidades subjetivas construidas en la investigación, las cuales } \\
\text { varían en su forma y contenido entre individuos, grupos y culturas. Por ello, el } \\
\text { investigador cualitativo parte de la premisa de que el mundo social es "relativo" y } \\
\text { sólo puede ser entendido desde el punto de vista de los actores estudiados. Dicho } \\
\text { de otra forma, el mundo es construido por el investigador. }\end{array}$ \\
\hline $\begin{array}{l}\text { Naturaleza de la } \\
\text { realidad }\end{array}$ & $\begin{array}{l}\text { La realidad no cambia por las observaciones } \\
\text { y mediciones realizadas. }\end{array}$ & La realidad sí cambia por las observaciones y la recolección de datos. \\
\hline Objetividad. & Busca ser objetivo. & Admite subjetividad. \\
\hline $\begin{array}{l}\text { Metas de la } \\
\text { Investigación }\end{array}$ & $\begin{array}{l}\text { Describir, explicar y predecir los fenómenos } \\
\text { (causalidad). } \\
\text { Generar y probar teorías. }\end{array}$ & $\begin{array}{l}\text { Describir, comprender e interpretar los fenómenos, a través de las percepciones y } \\
\text { significados producidos por las experiencias de los participantes. }\end{array}$ \\
\hline Lógica. & $\begin{array}{l}\text { Se aplica la lógica deductiva. De lo general a } \\
\text { lo particular (de las leyes y teoría a los datos). }\end{array}$ & $\begin{array}{l}\text { Se aplica la lógica inductiva. De lo particular a lo general (de los datos a las gener- } \\
\text { alizaciones -no estadísticas- y la teoría). }\end{array}$ \\
\hline $\begin{array}{l}\text { Relación entre cien- } \\
\text { cias físicas/naturales } \\
\text { y sociales. }\end{array}$ & $\begin{array}{l}\text { Las ciencias físicas/naturales y las sociales } \\
\text { son una unidad. A las ciencias sociales } \\
\text { pueden aplicárseles los principios de las } \\
\text { ciencias naturales. }\end{array}$ & $\begin{array}{l}\text { Las ciencias físicas/naturales y las sociales son diferentes. No se aplican los mis- } \\
\text { mos principios. }\end{array}$ \\
\hline
\end{tabular}

mejoramiento de la realidad. Es la imaginación transformativa. En la primera línea de su "Metafísica", Aristóteles (384 A.C.) dice que "Allmen by nature desire to know". Otros filósofos han llegado a complementar su idea al afirmar que el ser humano tiene una predisposición natural hacia la Metafísica, Kant, por ejemplo, la calificó de "necesidad inevitable"; Schopenhauer incluso definió al ser humano como "animal metafísico".

Desde la perspectiva de la epistemología (Piscoya, 2009), mayormente aplicada en las ciencias naturales (biología, física, etc.), la metafísica vendría a constituir la primera etapa en la concepción de un principio o teoría científica. Es la etapa auroral de una aventura científica que alcanza sólo hasta la formulación de una hipótesis. A partir de esta etapa comienza el protocolo de pruebas de laboratorio, de análisis estadísticos hasta aceptar la mencionada hipótesis como verdadera o descartarla como falsa.

\section{Los grandes metafísicos según Karl Jaspers}

Según Jaspers (1998), los mayores filósofos metafísicos de la humanidad han sido Anaximandro, Eratóstenes, Platón, Aristóteles, Parménides, Heráclito, Plotino, Anselmo, Spinoza, Lao Tse y Nagarjuna. Cada uno de ellos ha marcado una época y una forma de pensar en su tiempo y en su región, tanto en asuntos científicos como en asuntos cotidianos, es decir han marcado un paradigma, para usar el término de Kuhn (1962).

\section{TIPOS DE METAFÍSICA}

Si bien el avance del razonamiento humano sigue un mismo patrón (observación - abstracción - formulación), la metafísica (creatividad) se expresa en diversas formas, según el campo de aplicación. Una breve clasificación podría ser la siguiente: religiosa, normativa, científica, literaria, matemática, sociológica, entre otras. Veamos un poco de cada una de ellas.

- Metafísica religiosa. Todas las sociedades, a lo largo de la historia, han tenido y tienen su propia metafísica religiosa, es decir, su imaginario sobre el destino del hombre, de lo que ocurrirá después de la muerte. Ello ha permitido concebir en las principales religiones mundiales conceptos y figuras como cielo, infierno, pecado, ángeles, demonios, juicio final, etc. La metafísica religiosa es siempre dogmática y, en el más riguroso de los casos, sólo acepta el análisis de su consistencia lógica entre sus principios, lo que da origen, por ejemplo, al derecho canónico, ya que no es 
posible contrastar sus conceptos o problemas con ninguna parte de la realidad. Aquí no es importante el razonamiento sino la fe.

- Metafísica normativa. Fuente del derecho, la ética y la política. Ha sido desde el comienzo de la historia la primera manera de guiar a la sociedad, sobre la base de principios o mandamientos que todos deben cumplir para proteger a la especie y a su modo de vivir, es decir, a su cultura. La metafísica normativa está constituida por "visiones" de los líderes de la sociedad que buscan guiar la conducta humana por senderos "razonables". La metafísica normativa ha llegado a tener dos productos esenciales en las sociedades modernas: la ciencia del derecho y la ética. El derecho constituye la guía de toda sociedad moderna. En la mayoría de los países con sistemas legales "estatutarios", es parte de la Constitución del país que da origen a sus leyes, reglamentos $\mathrm{y}$ al conjunto de instituciones regulatorias que emanan de dicho ordenamiento jurídico. La ética, por su parte, está constituida por códigos de conducta individual captable o deseable que los individuos aceptan explícita o implícitamente como guías de su comportamiento personal por encima de las leyes. El comportamiento ético (no escrito en normas) es el modo de llegar a ser aceptado por una sociedad o grupo social determinado.

- Metafísica científica. Es la madre de la ciencia. Anaximandro es considerado el primer y más potente metafísico científico, allá por el siglo VIII A.C. Por ella se definen conceptos, se perfilan problemas, se configuran hipótesis, se diseñan "constructos" verificables. Las hipótesis, luego, siguen un riguroso proceso probatorio -basado en un inicio- en pruebas conceptuales y lógicas, para concluir con pruebas estadísticas. La inferencia estadística es el filtro de todo principio aceptado en la "ciencia dura". Esto ha sido denominado epistemología. Las hipótesis ya probadas dan nacimiento a las "leyes" o "principios" y la aplicación material a la solución de problemas concretos a la tecnología.

Desde el Renacimiento, y con ayuda del pensamiento filosófico positivista de Auguste Comte, la metafísica científica ha constituido el mecanismo esencial de la construcción de nuevos conocimientos de la ciencia en general, y de las ciencias naturales en particular. Es pues el origen de la ciencia moderna y la tecnología. Es decir, la ciencia y los científicos puros no conciben otra manera de llegar a la verdad.

El avance de la ciencia, sin embargo, no es absoluto; es, de algún modo coyuntural y convencional. Por ejemplo, Copérnico -con su propuesta de que el sol es el centro del universo- se impuso sobre las soluciones ampliamente aceptadas de su tiempo (el principio de Ptolomeo de que la tierra era el centro del universo). Más tarde puede y seguramente va a surgir una nueva propuesta científica sobre el mismo tema que será razonablemente demostrada, y el antiguo principio será descartado o pasará a un segundo plano, al relicario $o$ al basurero. A esto se denomina "cambio de paradigma", como lo denomina el filósofo norteamericano Thomas Kuhn (1922-1996) (1998).

Entre los metafísicos científicos hoy considerados de mayor alcance tenemos a Stephen W. Hawking (2009), astrofísico inglés, originalmente autodenominado neopositivista (como Popper), que ha llegado a afirmar que la física y la ciencia en general han avanzado a tal velocidad que la filosofía se ha quedado muy atrás en el camino, y más aún, que en su concepto, la filosofía ha muerto. Y, por lo tanto, lo único que quedaría es la capacidad de razonamiento metafísico concreto que sirva para construir el devenir de la ciencia.

- Metafísica literaria. Una forma de conducir al pensamiento hacia niveles insospechados a lo largo de toda la historia ha sido la literatura. El hombre ha imaginado realidades nuevas y las ha plasmado en obras detalladas que han servido de entretenimiento, $y$ muchas veces de inspiración a la ciencia. Por ejemplo, los conceptos "televisor" o "robot" han sido creados y así denominados por literatos mucho antes que la ciencia pudiera siquiera concebirlos como elementos viables

Nos atrevemos a afirmar que existen tres tipos de metafísica literaria: la utópica o de ilusiones, de fantasías, (como en la "Utopía" de Tomás Moro); la ucrónica, de ciencia ficción que construye realidades para otros tiempos, así tenemos a "Quo Vadis" o "In the year 2889" de Julio Verne; o que reconstruye otras como si en la realidad no se hubiera dado un hecho, por ejemplo que nunca se hubiera dado la Segunda 
Guerra Mundial. Finalmente tenemos a la metafísica literaria distópica que, al contrario de la metafísica utópica, es más bien producto de una imaginación derrotista y pesimista sobre la naturaleza del hombre. El mejor ejemplo es tal vez "1984" de Orwell.

- Metafísica matemática. La matemática es una ciencia formal, por lo tanto, producto de la observación, de la imaginación y del razonamiento. Los primeros metafísicos matemáticos fueron los asirios, babilonios, egipcios, mayas y árabes. De esta acumulación de sabiduría matemática surgieron la aritmética, el álgebra oral, luego la geometría, y la trigonometría de los griegos. El movimiento pitagórico, que duró muchos siglos, fue una secta matemática que fue trasladando este legado de generación en generación hasta llegar a una formulación formal (Bell, 1996). Ya en el periodo feudal los árabes nos obsequiaron con dos grandes herencias: el álgebra y la notación decimal. En esa misma época los hindúes dejaron su huella metafísica con el invento del número cero (0).

Luego, ni bien la humanidad salía de la Edad Media, el gran genio metafísico de Descartes creó la notación matemática que se utiliza desde entonces para expresar cualquier idea o problema de orden matemático en una sola línea. Además de la notación, el colosal invento del plano cartesiano (base de los análisis económicos y estadísticos) ha permitido mejorar el razonamiento de las mentes en los negocios. Desde entonces, virtualmente no existe problema empresarial que no pueda ser expresado en tres maneras alternativas: literaria, matemática y gráfica. Luego de Descartes, cualquier persona con inteligencia normal ha sido y es capaz de generar nuevas ideas con el soporte de la metafísica matemática.

El siglo XIX ha sido sin duda alguna el siglo de las mayores contribuciones matemáticas en la humanidad. Allí surgieron el genio de Gauss, cuyas contribuciones con el cálculo de probabilidades han hecho posible la evolución de las organizaciones y los negocios a su volumen actual. Luego Lovatchesky demostró que la geometría es un asunto de paradigmas, y que Euclides había sido el creador de una ciencia que puede tener muchas versiones, según los postulados en que se basen. Con Lovatchesky nacieron las geometrías no euclidianas y la relatividad plena en la creación de esta ciencia formal.
No hay dudas de que las matemáticas y sus infinitas aplicaciones tienen reservadas grandes sorpresas al futuro de la ciencia de la administración.

- Metafísica sociológica. Es la interpretación del mundo de todas las maneras posibles. Esta ha sido la metafísica utilizada masivamente por los pensadores sociales.

Su principal característica es que sigue la lógica siguiente:



Antiguamente los historiadores consideraban como su trabajo el reseñar con acuciosidad y objetividad los hechos ocurridos en el devenir de los pueblos, es decir su tarea era describir. Karl Marx (1818-1883) fue unos de los primeros sociólogos en cambiar este pensamiento al proponer un primer conjunto de leyes de la historia en una teoría llamada "Materialismo Histórico", que resulta ser una aplicación particular del concepto de dialéctica Hegeliana. Según Marx (Marx, 1848), tarde o temprano la sociedad evolucionaría dejando atrás el modo de producción capitalista abriendose las puertas al modo social comunista. Pero otros pensadores como Popper (1957), consideran más bien de muy mal gusto tratar de construir leyes sociales sobre la base del historicismo. Él sostiene que los hechos no se repiten.

No obstante, fue a partir de Emile Durkheim (1858-1917) (1982), padre de la sociología, que los pensadores sociales no solamente hacen descripciones detalladas de la realidad sino que las organizan en propuestas relacionales. La sociología moderna es exitosa y constituye la base del accionar de la política moderna, dejando un poco de lado al derecho. Es que se han llegado a construir una infinidad de modelos de tipo sociológico que explican cómo se comportan y evolucionan los grupos sociales. Estas teorías tienen mucho éxito, por ejemplo, cuando se dedican a explicar el origen de los conflictos sociales. Fue a partir de mediados del siglo XIX cuando muchos científicos sociales se basaron en la metafísica sociológica para construir sus teorías. Entre los influyentes sociólogos del siglo XX tenemos a Mc Luhan, Inner, Popper, y últimamente a Toffler y Fukuyama, entre otros. 
Particular mención en su calidad de gran metafísico de la naturaleza del ser humano merece Sigmund Freud, quien en el siglo XIX formuló importantes teorías que explican la conducta del individuo. Como toda teoría inteligente, los aportes de Freud causaron un enorme impacto en su momento, pero luego han devenido en poco creíbles, otras impopulares, inclusive algunas propuestas suyas son ahora consideradas como pura charlatanería metafísica, como la teoría de la interpretación de los sueños.

Modelo de metafísica de la ciencia de la administración

La administración es "el proceso de planear, organizar dirigir y controlar el empleo de los recursos organizacionales para alcanzar determinados objetivos de manera eficiente y eficaz" (Chiaventato, 2004). Es la disciplina dedicada al logro de resultados en una organización, a la ejecución. Es la disciplina de la eficacia con eficiencia (Drucker, 1974), es decir, de hacer las cosas que tocan hacer y de hacerlas con el mínimo uso de recursos. Este sentido pragmático y objetivo de la disciplina ha sido y es el fundamento de la permanente búsqueda "omnívora" en todos los campos, de todo tipo de medios sean reglas, modelos, principios o supuestos que permitieran arribar a resultados concretos de carácter efectivo.

Los pensadores de la administración no se limitan al razonamiento normativo o especulativo, o a aquel enmarcado en algún tipo de patrón metafísico. La evidencia está en que, a lo largo de su breve historia, han echado mano a todo tipo de ayuda filosófica, científica o tecnológica que permitiera inyectar eficacia y eficiencia a las organizaciones. Esta esencia pragmática, sincrética y teleológica les otorga la licencia para hacer uso de diversas herramientas de naturaleza y de origen científico o empírico variados; por lo tanto, se abre el camino para hurgar en todo tipo de combinaciones metafísicas.

En forma tentativa podríamos afirmar que esta búsqueda ha conducido a la ciencia de la administración a constituir su propio cuerpo de búsqueda o protocolo epistemológico que llamaríamos "metafísica de la ciencia de la administración", la que resulta del uso simple o combinado de una o más de las siguientes metafísicas: normativa, matemática, sociológica y sistémica. Veamos su valor o uso a lo largo de la generación de las teorías más conocidas y utilizadas en la ciencia de la administración (ver figura $\mathrm{N}^{\circ} 1$ )

Figura $\mathrm{N}^{\circ} 1$ El modelo de metafísica de la ciencia de la administración

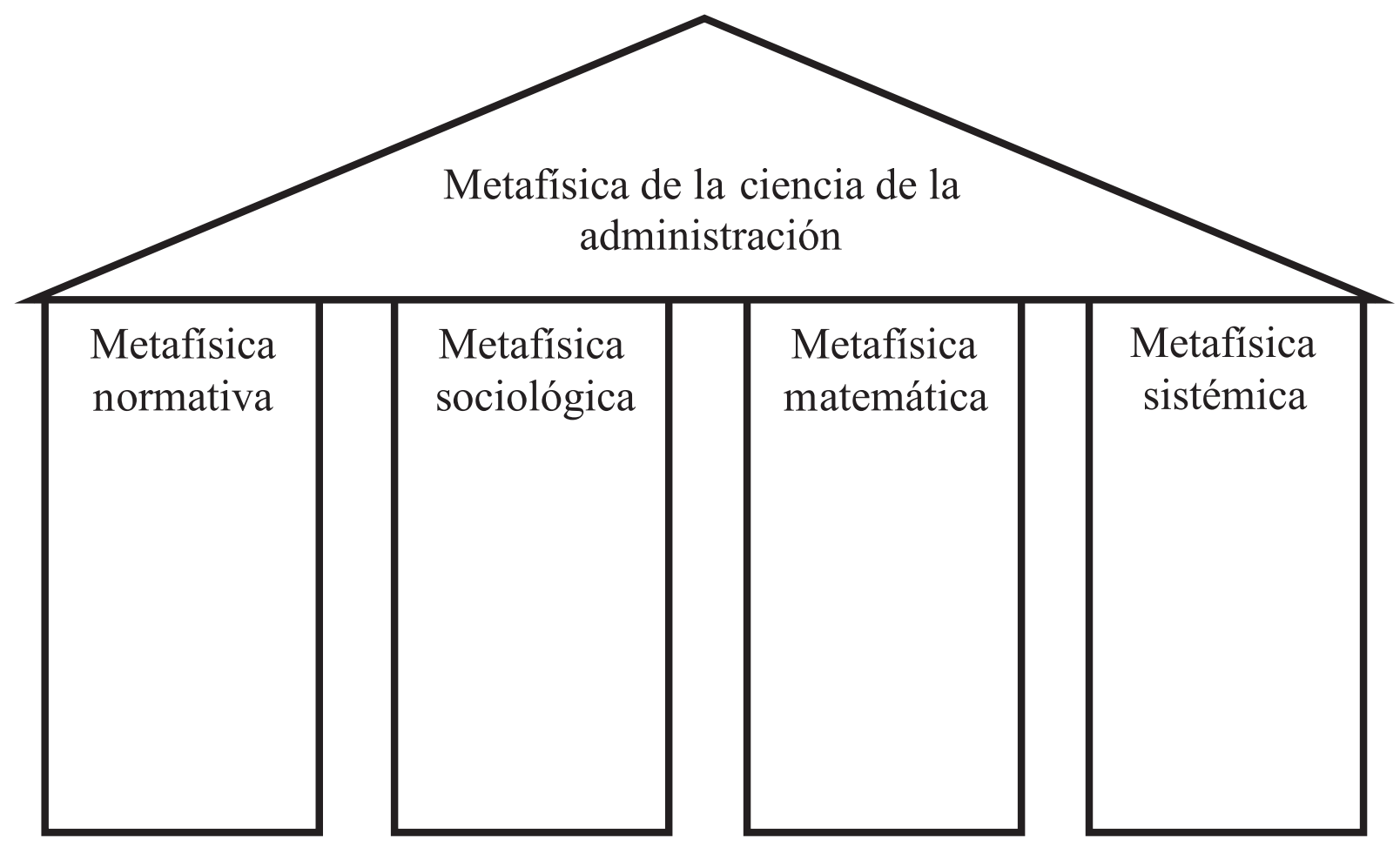


- Metafísica normativa en la administración. El primer impulso de todo gerente es mejorar el desempeño administrativo de la organización que maneja, por lo tanto, el instinto del razonamiento le aconseja formular principios generales de conducta aplicando la lógica siguiente:

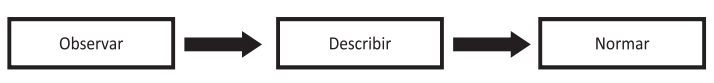

Las teorías administrativas pioneras tuvieron su origen en un esfuerzo metafísico normativo. Por este proceso nacieron las teorías clásicas. Entre ellas tenemos a las teorías de Fayol, Taylor, Gilbreth, Weber, etc. Veamos la primera.

\section{- Teoría de la organización clásica de} Henri Fayol.

Henri Fayol (1841-1925) ha generado tal vez la principal teoría de la ciencia de la administración, que sigue vigente con pequeños ajustes, por lo que la han llevado a ser denominada "Teoría Neoclásica de la Administración". Se afirma que Fayol de declaró positivista, en la tradición del también francés Auguste Comte. La herencia de Fayol está constituida por un conjunto de conceptos, modelos y principios que permitieran gobernar una organización. Estos elementos se pueden agrupar en tres grandes componentes: las funciones empresariales, funciones administrativas, y los principios administrativos.

Las funciones empresariales son seis y cubren las actividades esenciales de toda organización productiva: función comercial o de ventas, función técnica o de producción, función financiera, función contable, función de seguridad, y función administrativa (que controla a todas las demás).

Figura $\mathrm{N}^{\circ} 2$ El Modelo de funciones empresariales de Fayol



Las funciones administrativas son cinco y resumen el trabajo de un gerente: planeación, organización, dirección, coordinación y control. La siguiente figura puede mostrar la idea central de Fayol.

Figura $\mathrm{N}^{\circ} 3$ El Modelo de funciones administrativas de Fayol

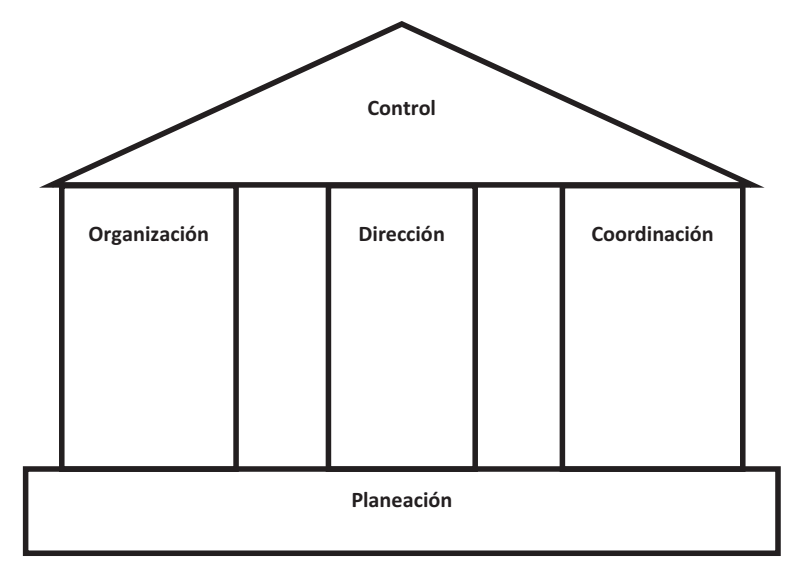

Los principios administrativos fueron 14 , sin embargo Fayol, con la honestidad monumental que lo caracterizó, señaló que investigaciones posteriores podrían incrementar o disminuir dichos principios: división del trabajo, autoridad y responsabilidad, disciplina, unidad de mando, unidad de dirección, subordinación del interés particular al general, remuneración del personal, jerarquía, orden, equidad, estabilidad del personal, iniciativa, unión del personal, y centralización.

Estas normas de conducta fueron propuestas en su libro "Administración Industrial y General" (Fayol, 1923) el cual constituye un manual de buenas prácticas gerenciales. Lo que se sabe es que Fayol, un ingeniero de minas, trabajó estos temas de manera individual y su único laboratorio y mecanismo epistemológico fue su poderosa inteligencia que le permitió capturar la realidad y expresarla en simples "mandamientos" para ser seguidos de manera creativa, nunca dogmática, por todas aquellas personas que estuvieran al frente de organizaciones.

Como observación histórica se puede afirmar que Fayol nunca se desenvolvió bajo el protocolo epistemológico de la ciencia dura, sino que su principal instrumento fue su capacidad de observación y de síntesis de la realidad. Esa poderosa capacidad me- 
tafísica de abstracción le permitió construir conceptos, categorías, luego principios, y finalmente relaciones entre ellas hasta llegar a generar los modelos explicados.

Similarmente, en la misma época pero en los Estados Unidos, Frederick Taylor propuso su "Teoría de la Administración Científica", que es así como él la denominó, que ha seguido en su génesis un derrotero metafísico muy similar al de Fayol. Taylor fue un gran asesor de empresas, y precisamente sus observaciones le llevaron a sintetizar las herramientas gerenciales en un conjunto de método para volver más eficiente cualquier trabajo sin importar si su naturaleza es de fábrica o administrativa.

- Modelo de organización burocrática. Otro caso de metafísica normativa en la ciencia de la administración lo constituye el modelo burocrático de Max Weber. Del mismo modo que Fayol y Taylor, Weber formuló un conjunto de mandamientos o principios que deben seguir las gerencias en general, sin exclusión alguna, para perfeccionar el funcionamiento de cualquier tipo de organización hasta llevarla a su mayor eficiencia y a sostenerse en el tiempo de manera indefinida. El objetivo de Weber fue crear un modelo de "organización ideal", independizada de sus dueños, perfecta y eterna.

Los principios de esta organización ideal fueron: especialización; funcionamiento basado en normas y reglamentos; existencia de una carrera administrativa; profesionalización y capacitación permanente; impersonalidad de las relaciones, y comunicaciones escritas.

Este modelo ha sido adoptado por las grandes organizaciones en el mundo, sea de tipo privada pero preferentemente las de naturaleza pública, siempre y cuando tuvieran un quehacer repetitivo y que tuvieran un entorno predecible.

Cabe mencionar como detalle histórico que previamente a la formulación de su modelo, Weber había publicado en 1905 el libro "La Ética Protestante y el Espíritu del Capitalismo" (Weber, 1985). Es decir, ya tenía experiencia en formulaciones sociológicas en el campo de la economía y de la empresa.
En los tres casos, Fayol, Taylor y Weber, la génesis de sus teorías se basó en una gran capacidad de observación y de abstracción de la realidad. Estos elementos estructurales no necesitaron de pruebas epistemológicas para demostrar su corrección; en su aplicación fueron demostradas ser verdaderas.

Luego del paso de un siglo, naturalmente que la aplicación de dichos modelos de gestión ha devenido en debatible, no por su naturaleza en sí sino porque el entorno en que viven las organizaciones ha cambiado radicalmente. Dichos modelos de gestión siguen funcionando pero con gran cantidad de ajustes.

- Metafísica sociológica en la administración. El método metafísico de la sociología ha servido desde un inicio para construir "conceptos", "categorías" y "modelos" que permitan explicar, y muy accesoriamente predecir el comportamiento de los grupos humanos en las organizaciones, aplicando el siguiente proceso:

\section{Explorar}

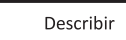

Teorizar

Los ejemplos más patentes de uso de metafísica sociológica lo constituyen la teoría de las relaciones humanas de Elton Mayo; la teoría de la autoridad de Max Weber; la jerarquía de las necesidades de Maslow, entre otras muchas teorías que habitan dentro de la llamada "escuela del comportamiento humano" o "comportamentista" de la administración.

- Teoría de las relaciones humanas de Elton Mayo. Cuando la aplicación del modelo de Taylor en Estados Unidos llegó a generar tal cantidad de disfunciones, la Universidad de Harvard encargó al psicólogo Elton Mayo (1880-1949) para que realice un conjunto de estudios sobre las razones de la variabilidad de la productividad en las empresas. El estudio que se realizó a fines de la década de los 20 se realizó en la fábrica Hawthorne de la empresa Western Electric. Después de cinco años y un holgado financiamiento, la conclusión fue que ningún aspecto material afecta tanto la producción de los trabajadores como el trato humano hacia ellos.

Mayo concluyó que en toda organización los trabajadores tienen dos tipos de comportamiento: formal e informal. El comportamiento formal es el que hace que el 
trabajador cumpla las reglas del contrato de trabajo, realice sus tareas y gane su salario, es el que hace que funcione la organización expresada en el organigrama y que hace que se cumplan los planes. El comportamiento informal es innato a la naturaleza humana, es el desfogue del ser humano como individuo. Se expresa en sus amistades, en sus preferencias, en su lenguaje en sus afectos y sus temores, en sus amores y odios, en sus expectativas y en sus frustraciones. $\mathrm{Si}$ la empresa es capaz de comprender esta faceta del trabajador, entonces es probable que la empresa se beneficie con una mayor productividad, mayores iniciativas, con mayor vocación de servicio. El modelo se expresa en la figura $\mathrm{N}^{\circ} 4$.

Figura $\mathrm{N}^{\circ} 4$ El modelo de organización formal e informal de Elton Mayo

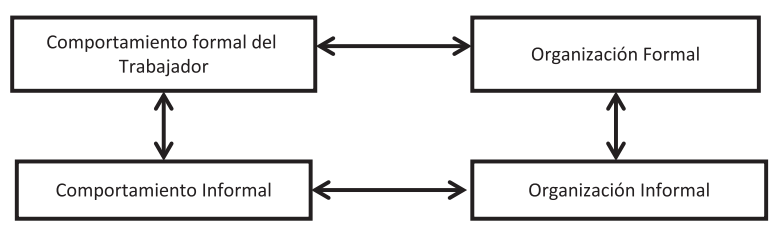

Falta concluir diciendo que este modelo tampoco nunca fue validado estadísticamente, sino que es producto de la intuición sociológica, y en realidad, fue adoptado, como ya se dijo, después de descartar todas las otras hipótesis que se probaron siguiendo el protocolo epistemológico universitario de entonces.

- Teoría de la autoridad de Max Weber. Esta teoría explica y predice por qué razones las personas obedecen en diferentes tipos de sociedades. Primero afirma que en el mundo existen esencialmente tres tipos de sociedades: tradicional, carismática y moderna. Luego cada uno de estos tipos de sociedad se ha convertido en una fuente correspondiente de tipo de autoridad. Entonces explica que si bien existen diversos tipos de sociedades, solamente hay un tipo de autoridad que dentro de una organización puede garantizar estabilidad en el largo plazo, en la ejecución de las órdenes y en el mantenimiento de la estructura y el orden interno. Esta es la autoridad legal o burocrática.

No niega que existan otros tipos de autoridad, particularmente la carismática que ejercen los líderes, pero la autoridad generalizada tiene que ser la de tipo legal que nace de un reconocimiento explícito y producto de un instrumento legal de la autoridad del mandante, particularmente si no es propietario de la organización, situación generalizada en el sector público. Para el caso de los "Tipos de Autoridad" de Weber, huelgan las explicaciones sobre la génesis absolutamente sociológica de su modelo (Litterer, 1998).

- La jerarquía de necesidades de Maslow. Según Abraham Maslow (1908-1970), las necesidades humanas son infinitas y se pueden agrupar en una escala de cinco niveles que vá de lo simple a lo complejo.

El primer nivel de las necesidades, el más elemental, está dado por las necesidades fisiológicas (comida, bebida, alojamiento, sexo, etc.), todos tenemos estas necesidades. El segundo nivel está dado por las necesidades de seguridad. Los seres humanos tenemos una natural aversión al riesgo, por lo tanto sentimos necesidad de seguridad en el trabajo, en las relaciones personales, etc. El tercer nivel lo constituye la necesidad de afiliación o de pertenencia, lo que quiere decir que las personas somos esencialmente gregarias, tenemos necesidad de pertenecer a grupos y queremos ser considerados parte de ellos. El cuarto nivel está dado por el ego, por el cual nos sentimos con derecho a una individualidad, queremos siempre ser reconocidos como protagonistas de algo en la vida, queremos que nuestras contribuciones y esfuerzos sean aceptados socialmente. Finalmente, el quinto nivel de necesidades se denomina de autorrealización, por el cual las personas tenemos en el fondo necesidades de justificar nuestro paso por este mundo, queremos dejar una contribución a la raza humana.

La teoría de Maslow sostiene que cada individuo, de acuerdo a su nivel económico, a su nivel cultural y a su nivel social experimenta una particular y única combinación de necesidades; lo que quiere decir que una persona en el trabajo estará satisfecha en la medida que el ambiente le ayude a satisfacer su escala de necesidades. Esta teoría constituye la piedra fundamental de la motivación laboral en las organizaciones. 
Por la complejidad y sutileza de esta teoría es extremadamente difícil siquiera imaginar que hubiera pasado por algún tipo de proceso epistemológico o de validación estadística. Sin embargo, esa omisión no le quita en absoluto mérito alguno. Su validez y confiabilidad radican en la interpretación que cada administrador haga para cada caso particular de aplicación.

En conclusión, las teorías administrativas relacionadas con el comportamiento de las personas nacieron, como se puede observar, de inspiración metafísica sociológica más que de ninguna prueba experimental o de laboratorio. Su aceptación en la ciencia de la administración fue el producto del grado de aplicabilidad que los gerentes les encontraron en la práctica cotidiana. Tal vez nunca hubieran alcanzado a tener categoría científica si se hubieran circunscrito a prácticas privadas pero, como estas ideas desde un inicio fueron de escrutinio público, es que llegaron a ser aceptadas y discutidas en todos los foros de estudios de la ciencia de la administración.

- Metafísica matemática en la administración. La metafísica matemática ha venido sirviendo de enorme instrumento para la construcción del cuerpo de conocimiento de la ciencia de la administración. Las disciplinas matemáticas puras, en su gran mayoría, han sido formuladas de manera teórica y sin un propósito particular. Han sido los académicos de la administración los que primero han problematizado su aplicabilidad para luego seguir el protocolo general de la epistemología de la ciencia (Maletta, 2009).

Entre los mayores metafísicos matemáticos de la administración se encuentra Luca Pacioli, un fraile que vivió en el siglo XV, quien fue el creador del principio matemático de la partida doble, principio sine qua non de la contabilidad. Sin embargo, no se conoce a ciencia cierta cuál habría sido el antecedente matemático de la contribución de Pacioli.

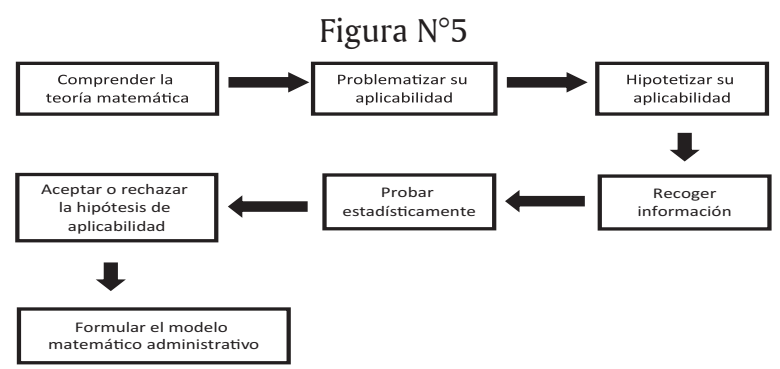

La contabilidad es una disciplina esencial para el control patrimonial y financiero de todas las organizaciones privadas o públicas. Constituye la base de la tributación empresarial y, por lo tanto, la base del funcionamiento de todos los estados modernos. Su esencia radica en el principio de la partida doble que establece que toda transacción económica, es decir que implique el movimiento de recursos medibles en términos monetarios, debe ser registrada en un doble asiento. Como resultado de ello, es posible elaborar diferentes cuadros de resultados de las operaciones conocidos con el nombre de estados financieros, los cuales permiten determinar si una empresa ha tenido ganancias o pérdidas en un periodo de tiempo dado. Los estados financieros más importantes son el balance general y el estado de ganancias y pérdidas.

Lo verdaderamente fantástico del uso del principio de la partida doble es que con las mismas cuentas usadas indistintamente para el registro de las operaciones (llamados asientos contables), es posible arribar a la misma cifra de resultado que es la ganancia o pérdida del período sea por la vía del balance general o del estado de ganancias y pérdidas. Esa es la esencia de la contabilidad. Esta creación es tan impresionante y mágica que Peter F. Drucker, uno de los padres modernos de la administración, la denominó el mayor invento en la administración de las organizaciones.

Otro gran metafísico matemático que contribuyó a la ciencia de la administración fue Adam Smith. Smith, escocés (1723-1790) considerado el padre de la economía. En su libro "La Riqueza de las Naciones" (Smith, 2012) demostró el principio de la división del trabajo, base de la productividad y de la eficiencia, sustento de la división social del trabajo dentro de las organizaciones, particularmente de aquellas que fueron incorporando los nuevos inventos tecnológicos de los siglos XVIII y XIX. La aplicación de dicho principio permitió pasar de la producción artesanal a la producción industrial, y a construir la sociedad moderna en que la humanidad vive. Hay que mencionar que la capacidad metafísica de Smith se había patentizado unos años antes cuando publicó el ensayo llamado "TheTheory of Moral Sentiments" (1759). Esto demuestra que Smith ya tenía ensayada su habilidad metafísica para construir modelos complejos. Esta obra de Smith compite plenamente 
con la "Teoría del hombre y teoría de la sociedad" de Thomas Hobbes. El principio de la división del trabajo demostrado por Smith sirvió posteriormente de base generalizada a los ingenieros pioneros que formularon las primeras propuestas de teorías administrativas como fueron Taylor y Fayol. El sociólogo Max Weber se unió al grupo.

A partir del alemán Carl Gauss (1777-1855), padre de la estadística y del cálculo de probabilidades, una inagotable fuente de inspiración surgió para enriquecer y dar precisión a la ciencia de la administración. A Gauss le siguieron otros sabios matemáticos de la segunda mitad del siglo XIX cuyos invenciones han servido de insumo para que un sinnúmero de científicos de la administración les encontraran decisivas aplicaciones en los campos del marketing, de las finanzas, del planeamiento y control de la producción, del control de la calidad, de los transportes, del almacenamiento, de los seguros, etc. que han servido para la minimización del riesgo de las inversiones, para mejorar la seguridad de los trabajadores y clientes, el uso óptimo de los recursos, así como para mejorar la calidad de los bienes y servicios producidos por los diferentes tipos de organizaciones de la sociedad moderna.

En este panorama, el muestreo aleatorio simple sigue constituyendo la base de la investigación de mercados y, por lo tanto, del pronóstico de las ventas y, consecuentemente, del cálculo y reducción del riesgo de las inversiones. De manera similar, el manejo adaptado de las distribuciones de probabilidades se ha convertido en la base de la matemática actuarial, base de la industria de los seguros. Como si eso fuera poco, las mismas herramientas matemáticas sostienen al análisis financiero y los complejos artilugios para el control de la calidad en la era digital.

Posteriormente en el siglo XX, particular mención corresponde el conjunto de formulaciones matemáticas realizadas en los laboratorios de combate durante la Segunda Guerra Mundial, muy en especial por los oficiales ingleses. Por ejemplo, el algoritmo matemático de la programación lineal

Cuadro $\mathrm{N}^{\circ} 2$ Principales teorías y modelos matemáticos y sus aplicaciones en administración

\begin{tabular}{|c|c|}
\hline Disciplina o modelo matemático & Modelo aplicado en la ciencia de la administración \\
\hline Aritmética & $\begin{array}{l}\text { Cálculo de la TIR (Tasa Interna de Retorno), VAN (Valor } \\
\text { Actual Neto), EBITDA. }\end{array}$ \\
\hline Álgebra & $\begin{array}{l}\text { Cálculo del lote económico o } \\
\text { Cantidad económica de pedido }\end{array}$ \\
\hline Geometría analítica & $\begin{array}{l}\text { Análisis de costos y ventas } \\
\text { Análisis de sensibilidad y de punto de equilibrio } \\
\text { Modelos de pronósticos }\end{array}$ \\
\hline Análisis de Pareto & $\begin{array}{l}\text { Gestión de inventarios } \\
\text { Análisis de calidad }\end{array}$ \\
\hline Distribuciones de probabilidades & $\begin{array}{l}\text { Análisis actuarial } \\
\text { Industria de seguros y reaseguros } \\
\text { Modelos de calidad }\end{array}$ \\
\hline Programación lineal & $\begin{array}{l}\text { Reducción de desperdicios de materias primas en las in- } \\
\text { dustrias. } \\
\text { Generación de fórmulas de alimento balanceado para las } \\
\text { aves, porcinos, producción de leche, etc. }\end{array}$ \\
\hline PERT (Program Evaluation and Review Technique) & $\begin{array}{l}\text { Programación de producción. } \\
\text { Control de proyectos de construcción }\end{array}$ \\
\hline CPM (CriticalPathMethod) & Optimización de programas de producción \\
\hline Teoría del muestreo & $\begin{array}{l}\text { Investigación de mercados } \\
\text { Estudios de la opinión pública. } \\
\text { Análisis de calidad. }\end{array}$ \\
\hline Análisis de regresión simple y múltiple & Pronósticos de la empresa, la industria y el país \\
\hline Árboles de decisión & Optimización de decisiones \\
\hline
\end{tabular}


creado en 1947 por los ingleses George Dantzig y John von Neumann de la formulación general siguiente:

$$
f=C_{1} X_{1}+C_{1} X_{1}+C_{1} X_{1}+\ldots+C_{1} X_{1}
$$

Fue adoptada inmediatamente en los negocios para la reducción de desperdicios en las industrias modernas, la creación de fórmulas alimenticias en las industrias de la crianza de animales y de producción de leche.

El cuadro $\mathrm{N}^{\circ} 2$ intenta resumir algunas de las teorías o disciplinas matemáticas que han servido de base para que los teóricos de la administración hicieran un uso de una colosal capacidad de inventiva metafísica para encontrarles aplicabilidad en el campo del mejoramiento de la gestión de las organizaciones y que, progresivamente, fueron incorporados al acervo de la actividad gerencial cotidiana.

La adaptación de las teorías matemáticas a la administración, como ya se dijo antes, ha seguido el protocolo epistemológico de la ciencia dura; es decir, han tenido que ser formuladas y probadas experimentalmente, para luego demostrar que su uso es viable y con el mínimo de riesgo.

- Metafísica sistémica. La Teoría General de Sistemas (TGS) surgió a fines de los años 40 en el marco de las ciencias naturales; primero con el modelo de Shannon y Weaver aplicado a la Electrónica, posteriormente con la propuesta de Ludwig von Bertalanffy aplicada a la biología. En su aplicación a las organizaciones, por ejemplo, la TGS ayuda a enfocarlas y comprenderlas como símiles de las células y los organismos vivos.

En general la TGS, con la ayuda de los principios generales de los sistemas abiertos, ha generado una nueva forma de razonar en la administración, de manera negentrópica, homeostática y holística. Una buena noticia adicional constituye el hecho que la TGS ha sido adoptada por las diversas ciencias, en general, como nueva plataforma de pensamiento. Así, mientras que en los dos últimos siglos la expansión de la ciencia ha significado la súper especialización de las ciencias, muchas veces de espaldas entre sí, la TGS convoca a un reentendimiento entre ellas; nueva situación que genera una ventaja enorme a la ciencia de la administración porque, siguiendo su propia dinámica, ahora está más cerca de las demás ciencias para observar aquello que pueda serle de utilidad.

Precisamente, una de las aplicaciones tecnológicas que está hermanando a todos los cultores del conocimiento, sin importar su especialidad, es la aplicación masiva de la informática. Pareciera que el nuevo lenguaje común de las ciencias resulta ser la computación sobre una plataforma de redes mundiales de fibra óptica. Esto hace que, como nunca antes, los conocimientos de diferentes campos estén al alcance de unos y otros. Esto es lo que está llevando a "pensar sistémicamente".

Así, mientras que los antiguos teóricos de la administración recurrieron a la ciencia del derecho, otros a las matemáticas, otros a la sociología como fuentes de conceptos, principios y modelos, hoy, existe la obligación de hurgar en otros campos, muy en especial en el campo de la investigación tecnológica de comunicaciones y de sistemas.

En este tema, la investigación académica en administración en los países desarrollados parece haber perdido el camino. La evidencia concreta es que las mayores empresas actuales del mundo son tecnológicas y están dedicadas al desarrollo de nuevos sistemas de comunicación basados en la ciencia de la Informática, como Microsoft, Apple, Google, Federal Express, etc., las mismas que, por coincidencia, han sido creadas por estudiantes que abandonaron las aulas universitarias al comprobar que sus centros de estudio no se alineaban con sus expectativas y sus inquietudes.

Desafíos del nuevo entorno de las organizaciones

La complejidad de las nuevas organizaciones y de su entorno ha hecho que la TGS sea la fuente de inspiración para comprenderlas y manejarlas. Pero, adicionalmente, es preciso reconocer que a partir de los años 90, el entorno y el interno de las organizaciones han cambiado de manera dramática en los siguientes vectores:

- Ha aumentado la velocidad de hacer negocios como resultado del crecimiento de los medios de comunicación mundial.

- Ha aumentado la dimensión y la complejidad de las organizaciones. Han surgido hiper- 
organizaciones como Walmart, con cerca de 850.000 trabajadores, frente a la cual, organizaciones también gigantescas como Federal Express, con más de 250.000 trabajadores, parecen más bien organizaciones medianas (Daft, 2011).

- Los mercados se han globalizado, ya no existen fronteras para los negocios. Casi se ha perdido el concepto de nacionalidad de las empresas. La competencia es imposible hasta de calcular.

- Ha aumentado exponencialmente la velocidad de la toma de decisiones como consecuencia del surgimiento de la informática y la computación desde los años 50.

- Ha surgido el teletrabajo, es decir el trabajo desde cualquier parte. Cada vez es menos necesaria la presencia física del trabajador.

- Ha disminuido la lealtad al trabajo, los trabajadores ahora se quedan un promedio de dos años en una empresa. Los trabajadores reclaman su propio derecho a la construcción de sus carreras.

- Cada día se vigoriza el sistema de ventas electrónicas tipo B2C (business to customers), por el cual los clientes ya no necesitan salir de sus casas para comprar.

- Las redes sociales se están convirtiendo cada vez más en un factor crucial en la aceptación de los productos y servicios.

- La gran sensibilidad global por el deterioro ecológico es cada vez mayor, lo que se ha convertido en una nueva y severísima restricción del trabajo gerencial. Por ejemplo, hoy no podríamos inventar el automóvil a petróleo, como ocurrió hace 100 años.

Las organizaciones cada vez más tienen miedo al futuro, nadie puede siquiera asegurar que sobrevivirán dentro de 10 años. Las teorías actuales

Cuadro No. 3. Las principales teorías administrativas y su fuente metafísica

\begin{tabular}{|c|c|}
\hline Fuente metafísica & Teoría o modelo de administración \\
\hline Metafísica normativa & $\begin{array}{ll}\text { - } & \text { Teoría de la Administración Científica de F.W. Taylor } \\
\text { - } & \text { Teoría Clásica de la Administración de H. Fayol } \\
\text { - } & \text { Teoría Burocrática de M. Weber (modelo normativo) } \\
\text { - } & \text { Administración por Objetivos (Peter Drucker) } \\
\text { - } & \text { ISO } 9001 \text { y los diversos modelos de sistemas de aseguramiento de la calidad. }\end{array}$ \\
\hline Metafísica sociológica & $\begin{array}{ll}\text { - } & \text { Teoría Burocrática de M. Weber (modelo de autoridad) } \\
\text { - } & \text { Teoría de la influencia religiosa sobre la administración de Max Weber. } \\
\text { - } & \text { Teoría de las Relaciones Humanas de E. Mayo } \\
\text { - } & \text { Teoría X y Y de Mc Gregor } \\
\text { - } & \text { Teoría de la Escala de las Necesidades de Maslow } \\
\text { - } & \text { Teoría de Skinner } \\
\text { - } & \text { Teoría Motivacional de Hertzberg } \\
\text { - } & \text { Teorías de liderazgo } \\
\text { - } & \text { Sistema de Calidad Total (TQM) }\end{array}$ \\
\hline Metafísica matemática & $\begin{array}{ll}\text { - } & \text { Aplicaciones de Programación Lineal } \\
\text { - } & \text { Modelos PERT, CPM. } \\
\text { - } & \text { Teorías de Colas para mejorar la atención en supermercados y bancos. Teoría } \\
& \text { de Montecarlo. } \\
\text { - } & \text { Teorías de Cadenas de Markov } \\
\text { - } & \text { Modelos de Asignación de Recursos } \\
\text { - } & \text { Modelos de Transportes } \\
\text { - } & \text { Arboles de Decisión } \\
\text { - } & \text { Modelos Bayesianos de decisiones } \\
\text { - } & \text { Modelos de Calidad. }\end{array}$ \\
\hline Nueva metafísica sistémica & $\begin{array}{l}\text { - Teoría de los Sistemas Administrativos } \\
\text { - } \quad \text { Teoría de las Contingencias }\end{array}$ \\
\hline
\end{tabular}

Fuente: Chiavenato, 2006.

Elaboración: Propia. 
en la administración han resultado insuficientes. Se requiere urgentemente de nuevos enfoques, por esta razón hay que promover la apertura del pensamiento en su máxima expresión. Hay que dar licencia a todo tipo de razonamiento científico con tal que ayude a comprender y resolver los nuevos problemas administrativos, que son los problemas de la sociedad.

\section{Conclusión sobre la metafísica de la ciencia de la administración}

La ciencia de la administración, al ser una ciencia nueva con algo menos de un siglo de vida, ha ido construyendo paso a paso su propia metafísica; es decir, su propio motor de progreso. Esto ha conducido a la administración a ir constituyendo un cuerpo implícito del conocimiento epistemológico propio, incorporando para sí por lo menos cuatro tipos de metafísica: normativa, sociológica, matemática y sistémica. Siguiendo a Chiavenato (2006), en el Cuadro $\mathrm{N}^{\circ} 3$ se hace un ensayo de recuento, limitado, y seguramente con grandes omisiones, sobre las fuentes metafísicas y los productos cognoscitivos concretos que hoy son parte del acervo de la ciencia de la administración.

Sin embargo, muchos de estos modelos administrativos se mantienen vigentes aún en su versión metafísica; es decir, en el estado de describir una propuesta hipotética de cómo funciona la realidad organizacional. Eso ocurre porque nunca han sido conducidos por el duro sendero de las pruebas epistemológicas; es decir, el ser probados estadísticamente y demostrar el grado de certidumbre que cada uno de dichos modelos tiene, lo que habría dado lugar al nacimiento de una "ley" o "principio". Ello no es raro o no es un error; lo que pasa simplemente es que eso no es posible. Por su complejidad sociológica o psicológica, esos modelos no pueden ser probados en laboratorio alguno. Esto hace que para algunos filósofos, como Mario Bunge (1960), la administración aún no adquiera la categoría de ciencia.

\section{Aplicación práctica de los resultados de la in- vestigación.}

Los resultados de este estudio deberían conducir a ampliar los métodos de investigación en la ciencia de la administración, de tal manera que se logre una lluvia permanente de teorías, modelos, principios o reglas, sin jamás albergar el temor a llegar a tener nuestros propios "herejes de la ciencia" (Polanco, 2003).
Así mismo, deben ayudar en un futuro a mejorar su enseñanza y, como consecuencia, a mejorar su ejercicio profesional. Como efecto inmediato se sugiere el cambio de los reglamentos de investigación académico para tesis de bachiller, maestría y doctorado. Este cambio debería abrir e incorporar el modelo de investigación cualitativa, adicional al ya existente modelo de investigación cuantitativa. En general, la investigación académica en administración debe promover el uso o ensayo de los cuatro tipos de metafísica (normativa, sociológica, matemática y sistémica), de tal manera que -indistintamente al uso instrumental del modelo de investigación cuantitativo o cualitativo- permita arribar a resultados utilizables en el quehacer gerencial. Naturalmente que para ello se deben definir los alcances, límites así como los mecanismos de evaluación de cada investigación en particular.

\section{CONCLUSIONES}

1. Las principales teorías de la ciencia de la administración han tenido su génesis en la creación cualitativa a partir de esfuerzos de los pioneros de construir un cuerpo de conocimientos que resuelva los problemas concretos de la gestión "aquí y ahora". Estas teorías primigenias han tenido su base en la metafísica normativa y la metafísica sociológica.

2. Posteriormente, a partir de la Segunda Guerra Mundial, los teóricos de la administración han recurrido cada vez más a las matemáticas, en cuyas fuentes del conocimiento se han inspirado para buscarles su adaptación a la realidad de las organizaciones y de los negocios. Su base ha sido la metafísica matemática, la misma que ha seguido el proceso epistemológico clásico.

3. Existe, de manera implícita, una metafísica de la ciencia de la administración que no es original sino de segundo piso, la misma que soporta a las metafísicas normativa, sociológica, matemática y, últimamente, a la sistémica.

\section{LITERATURA CITADA}

Aristóteles. (2012). Metafísica. Amazon Edición Kindle.

Bell, E.T. (1996). Historia de las Matemáticas. Fondo de Cultura Económica. México. 
Bernal, César. (2010). Metodología de la Investigación. Administración, Economía, Humanidades y Ciencias Sociales. Tercera Edición. Pearson - Prentice Hall, México.

Bonilla Castro, Elssy; Hurtado Prieto, Jimena y Jaramillo Herrera, Christian. (2009). La Investigación. Aproximaciones a la construcción del conocimiento científico. Alfaomega, México.

Bunge, Mario. (1960). La Ciencia, su método y su Filosofía. Editorial El Ateneo, Buenos Aires.

Campbell, Tom. (1985). Siete Teorías de la Sociedad. Colección Teorema. Sexta Edición. Editorial Cátedra. Madrid.

Chiavenato, Idalberto. (2006). Introducción a la Teoría General de la Administración. Séptima edición. Mc Graw Hill Interamericana, México.

Daft, Richard L. (2011). Teoría y Diseño Organizacional. Ediciones CENGAGE Learning, México.

Drucker, Peter. (2010). Management. Harper \& Row Management Library. Amazon. Kindle book. http://www.amazon.com/The-Practice-of-Management-book/dp/B003F1W$\mathrm{M} 8 \mathrm{E} / \mathrm{ref}=\mathrm{sr} 1$ _2?s = books\&ie $=\mathrm{UTF} 8 \&$ $\mathrm{q}$ i d $=1382234417 \& \mathrm{~s} \mathrm{r}=1$ 2\&keywords $=$ drucker + management

Durkheim, Emile. (1982). Las Reglas del Método Sociológico. Hyspamérica, Madrid.

Fayol, Henri. (1996). Administración Industrial y General. BCE El Ateneo Editores. Buenos Aires.

Hawking, Stephen W. (2009). Agujeros Negros. Santillana Ediciones. Madrid.

Hernández Sampieri, Roberto; Fernández Collado, Carlos y Baptista Lucio, María del Pilar. (2010). Metodología de la Investigación. Quinta Edición. McGraw Hill, México.

Herodoto. (1982). Los Nueve Libros de la Historia. Ediciones ORBIS. Hyspamérica. Madrid.

Jaspers, Karl. (1998). Los Grandes Filósofos. Editorial Tecnos, Madrid.
Jenofonte. (1963). “Economía”. En: Clásicos Jackson. W. M. Jackson Inc. Editores, México.

Kuhn, Karl. (1998). La Estructura de las Revoluciones Científicas. Tecnos, Madrid.

Litterer, Joseph. (1998). Análisis de las Organizaciones. Limusa, México.

Maletta, Héctor. (2009). Epistemología Aplicada: Metodología y Técnica de la Producción Científica. CEPES, Universidad del Pacífico, Lima.

Mc Gregor, Douglas. (1981). El Aspecto Humano de las Empresas. Diana, México.

Piscoya Hermoza, Luis. (2009). Tópicos de Epistemología. Fondo Editorial UIGV, Lima.

Polanco Massa, Alejandro. (2003). Herejes de la Ciencia. Corona Borealis. Madrid.

Popper, Karl. (1957). La Sociedad Abierta y sus Enemigos. Paidós, Barcelona.

Smith, Adam. (2012) The Wealth of Nations. Amazon. Kindle book. (http://www.amazon.com/ Inquiry-Nature-Causes-Nations-ebook/dp/ $\mathrm{B} 00847 \mathrm{CE} 60 / \mathrm{ref}=\mathrm{sr} 1$ 1_1 $\mathrm{s}=$ books\&ie $=\mathrm{U}$ TF8\&qid $=13822337 \overline{5} 7 \& \mathrm{sr}=1-1$ \&keywords $=$ adam + smith + the + wealth + of + nations

Taylor, Frederick W. (2012). Administración Científica. Amazon.Edición kindle. E-book. (http:// www.amazon.com/Principles-ScientificManagement-ebook/dp/B0082Y8IWS/ref=s r_1_1?s=books\&ie $=$ UTF8\&qid $=1382235$ $\overline{19} 1 \& \mathrm{sr}=1-1 \&$ keywords $=$ taylor + frederick.

Verne, Jules y Verne, Michael. (2013). In the year 2889. Amazon. Kindle edition. http://www. amazon.com/In-the-Year-2889-ebook/ $\mathrm{dp} / \mathrm{B} 0082 \mathrm{YX} 5 \mathrm{QM} / \mathrm{ref}=\mathrm{sr} 1$ __ 1 ?s = book $\mathrm{s} \& \mathrm{ie}=\mathrm{UTF} 8 \& \mathrm{qid}=13822 \overline{3} 8 \overline{1} 02 \& \mathrm{sr}=1$ $1 \&$ keywords $=$ verne +2889

Weber, Max. (1985). La Ética Protestante y el Espíritu del Capitalismo. Hyspamérica, Madrid.

Westernunion.com. (2012). "Our Rich History". Fecha de consulta: 10/07/2013. Disponible en: <http:/corporate.westernunion.com/ History.html $>$. 